Rev Inv Vet Perú 2015; 26(1): 146-151

http://dx.doi.org/10.15381/rivep.v26i1.10918

\title{
Comunicación
}

\section{Hematometra e Hiperplasia Endometrial Quística en una Perra: Descripción de un Caso}

\author{
Hematometra and Cystic Endometrial Hyperplasia in a Bitch: A Case Description
}

Alfonso Sánchez R. ${ }^{1,2}$

\section{Resumen}

\begin{abstract}
Se describe el caso de una perra Bullmastiff de 4.5 años que fue presentada a consulta por secreción vaginal hemorrágica prolongada. No se observó compromiso sistémico en el examen ginecológico, se estableció el estado de anestro por citología vaginal y medición de progesterona plasmática $(<1 \mathrm{ng} / \mathrm{ml})$, y se confirmó la presencia de contenido y aumento de tamaño uterino mediante ecografía. Además, se determinó una leve anemia regenerativa. Dado el historial de ciclos estrales irregulares de la hembra, se optó por la ovariohisterectomía como tratamiento. En la evaluación del útero se observó la presencia de sangre en el lumen uterino y de quistes en la mucosa, dándose como diagnóstico una hematometra concomitante con hiperplasia endometrial quística.
\end{abstract}

Palabras clave: hematometra; hiperplasia endometrial quística; perra

\section{Abstract}

The case of a 4.5 years old Bullmastiff bitch presented to the clinic with prolonged haemorragic vaginal discharge is described. A systemic involvement was not observed in the gynecological examination, the anestrus status was determined by vaginal cytology and measurement of the progesterone level in plasma $(<1 \mathrm{ng} / \mathrm{ml})$, and the presence of uterine content and increased uterine size was confirmed by ultrasonography. Besides, a mild regenerative anemia was determined. Based on a history of irregular estrous cycles an ovariohysterectomy was performed as treatment. In the evaluation of the uterus was observed the presence of blood in the uterine lumen and cysts in the mucosa, what led to the diagnosis of hematometra concomitant with cystic endometrial hyperplasia.

Key words: hematometra; cystic endometrial hyperplasia; bitch

\footnotetext{
${ }^{1}$ Escuela de Medicina Veterinaria, Universidad Santo Tomás, Viña del Mar, Chile

${ }^{2}$ E-mail: teriogenologiachile@vtr.net
}

Recibido: 13 de enero de 2014

Aceptado para publicación: 30 de abril de 2014 


\section{INTRODUCCIÓN}

La perra doméstica (Canis lupus familiaris) es una hembra monoéstrica, no estacional, polítoca, ovuladora espontánea, con una fase luteal de aproximadamente dos meses de duración, seguida de un anestro obligatorio de duración variable. El intervalo interestral (IIE) fluctúa entre los 5 y 12 meses, sin presentar diferencias significativas entre hembras gestantes y no gestantes (Concannon, 2011). Dicha ciclicidad puede verse alterada por la aparición de estructuras ováricas patológicas, tumores o quistes. Por otra parte, el estímulo progestagénico prolongado predispone a las perras a patologías uterinas; donde, además de los tumores que son de baja prevalencia, se destacan la piometra, la hiperplasia endometrial quística (HEQ) y la metrorragia (Fieni, 2006).

En la clínica canina, la patología uterina más común es la piometra, alteración descrita como una enfermedad diestral, de hembras adultas enteras, que se caracteriza por acumulación de pus en el útero y signos reproductivos, sistémicos, hematológicos e imagenológicos variables (Tello et al., 1996; Bigliardi y Pamigiani, 2004; Pretzer, 2008; Verstegen et al., 2008). La piometra también ha sido denominada piometritis, complejo piometra, endometritis catarral, endometritis purulenta, endometritis quística crónica, endometritis purulenta crónica, complejo endometritis-piometra y complejo hiperplasia endometrial quística/piometra; esta última denominación es la más utilizada (Johnston et al., 2001; Pretzer, 2008; Kempisty et al., 2013).

La HEQ de la perra es considerada como un trastorno reproductivo subclínico que afecta la fertilidad y se le ha asociado con respuesta anormal del endometrio a las hormonas ováricas. Se caracteriza por engrosamiento del endometrio, que ocurre por aumento en número y tamaño de las células epiteliales y de las glándulas endometriales (Schlafer, 2012). La prolongada estimulación hormonal durante la fase diestral del ciclo sexual de la perra condicionaría un incremento en el número y actividad de las células secretoras, con la consiguiente acumulación de exudados en el útero (Chen et al., 2001). Además, esta hiperplasia e hipertrofia glandular con hipersecreción de las glándulas endometriales facilitaría la formación de quistes con líquido estéril en el útero (Schlafer, 2012).

Según Fieni (2006), la metrorragia se caracteriza por presentar sangrado vulvar fuera del periodo del calor o celo. La hematometra o hemometra se define como la acumulación de sangre estéril en el útero, y ha sido descrita asociada a torsión uterina y a intoxicación con rodenticidas anticoagulantes (Johnston et al., 2001).

\section{Caso Clínico}

Una hembra Bullmastiff, nulípara, de 4.5 años, $47 \mathrm{~kg}$ de peso corporal y con historial de ciclos estrales irregulares (IIE <3-4 meses), es llevada a consulta de especialidad al Hospital Veterinario de la Universidad Santo Tomás, en Viña del Mar, Chile, por presentar secreción vaginal sanguinolenta los últimos 15 días, sospechándose de un estado de proestro prolongado.

En el examen clínico general, las constantes fisiológicas se encontraron dentro de los rangos normales para la especie, en tanto que a la palpación de abdomen, no se observó manifestación de dolor y el útero se notaba aumentado de tamaño. En el examen ginecológico se corroboró la presencia de abundante secreción sanguinolenta escurriendo por la vulva pero no se observó edema vulvar (Fig. 1a). Se obtuvo un hisopado vaginal para evaluación de la citología, donde destacó el predominio de células parabasales e intermedias pequeñas (>80\%) y abundantes eritrocitos. Se tomó una muestra de sangre de la vena cefálica para medir progesterona sérica mediante un kit comercial semicuantitativo (Ovulation ${ }^{\circledR}$ test), que dió como resultado una concentración $<1 \mathrm{ng} / \mathrm{ml}$. 

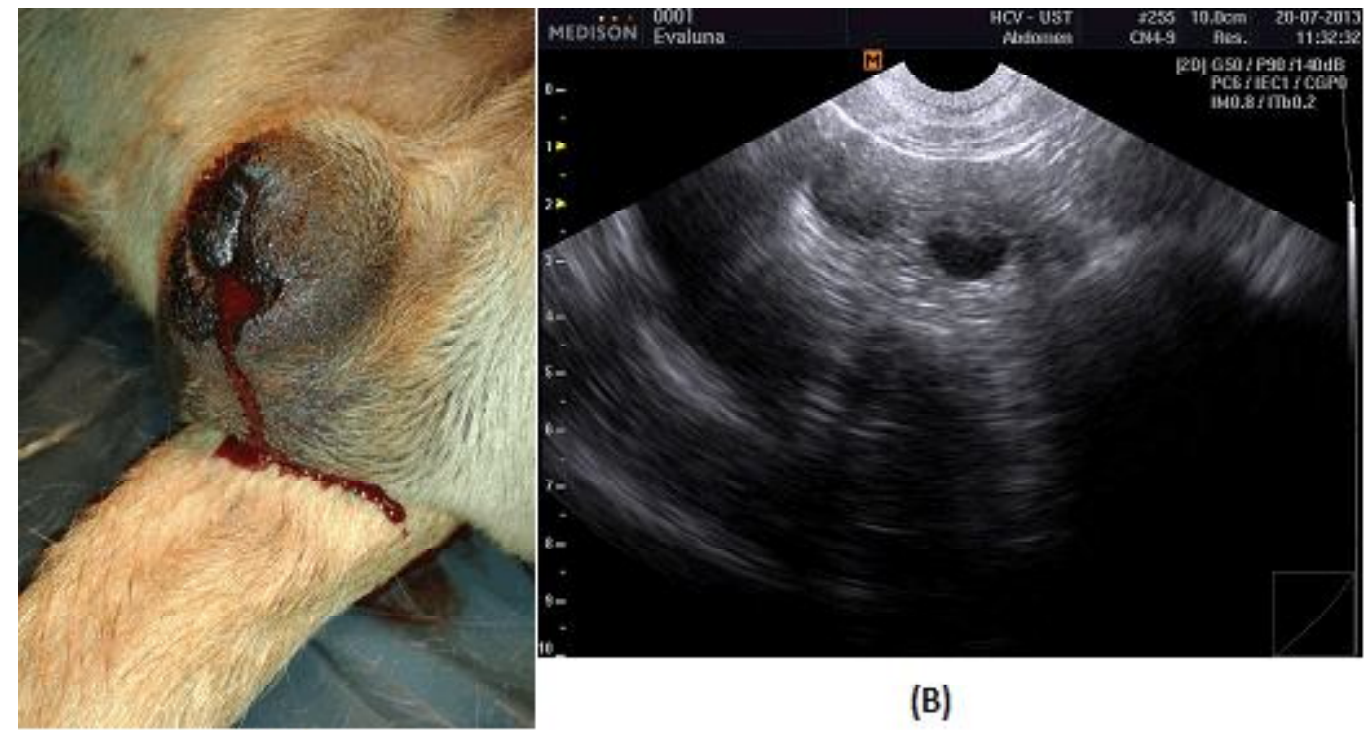

(B)

(A)

Figura 1. Perra Bullmastiff, nulípara, de 4.5 años, con historial de ciclos estrales irregulares, con secreción vaginal sanguinolenta por 15 días. (A) Vista de la zona vulvar con abundante secreción sanguinolenta y ausencia de edema vulvar; (B) Ecografía uterina donde se destaca un área central anecoica limitada por una pared ecogénica, característico de presencia de contenido.

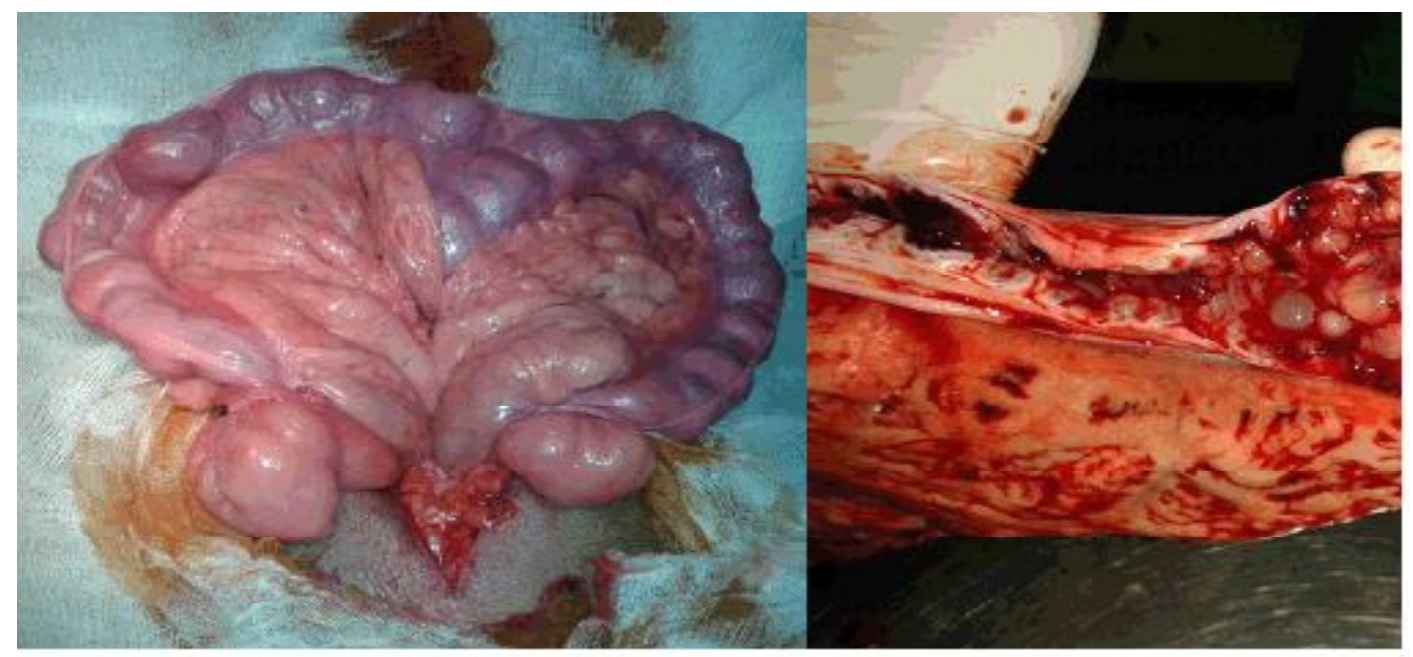

(A)

(B)

Figura 2. Perra Bullmastiff, nulípara, de 4.5 años, con historial de ciclos estrales irregulares, con secreción vaginal sanguinolenta por 15 días. (A) Útero vesiculado en forma de collar de perlas; (B) Vista dorsal de un cuerno uterino abierto longitudinalmente, donde destaca la presencia de sangre en el lumen y las lesiones quísticas en la mucosa 
Cuadro 1. Resultados del hemograma de una perra Bullmastiff, nulípara, de 4.5 años, con secreción vaginal sanguinolenta por 15 días

\begin{tabular}{lcc}
\hline Parámetro & Valor en el paciente & Valor de referencia $^{1}$ \\
\hline Hematocrito $(\%)$ & 35 & $37-55$ \\
Hemoglobina $(\mathrm{g} / \mathrm{dl})$ & 11.2 & $12-18$ \\
Eritrocitos $\left(\mathrm{x} 10^{6} / \mathrm{ml}\right)$ & 5.3 & $5.5-8.5$ \\
Leucocitos $\left(\mathrm{x} 10^{3} / \mathrm{ml}\right)$ & 11.6 & $6-17$ \\
Neutrófilos $(\%)$ & 70 & $65-73$ \\
Eosinófilos $(\%$ & 2 & $1-8$ \\
Linfocitos $(\%)$ & 13 & $9-26$ \\
Plaquetas $\left(\mathrm{x} 10^{3} / \mu \mathrm{l}\right)$ & 215 & $160-525$ \\
Reticulocitos $(\%)$ & 2 & $0-1.5$ \\
\hline
\end{tabular}

${ }^{1}$ Fuente: Jain (1993)

Según los hallazgos colpocitológico y hormonal se pudo concluir que la secreción vaginal sanguinolenta no estaba asociada a la actividad ovárica característica del periodo de calor de la perra, de modo tal que se descartó el estado de proestro y se confirmó la condición de anestro.

Con el propósito de establecer el origen del sangrado se realizó un examen ultrasonográfico, observándose el útero saculado como una zona anecogénica limitada por una pared ecogénica (Fig. 1b). El diámetro de los cuernos uterinos fluctuó entre 47 y $50 \mathrm{~mm}$. Asimismo, en forma complementaria, se realizó un hemograma donde se encontró anemia moderada (Cuadro 1).

Ante el negativo historial reproductivo de la hembra por sus ciclos irregulares y la evidencia del compromiso uterino, se optó por el tratamiento quirúrgico realizándose una ovariohisterectomía. Al extraer el tracto genital y observar las estructuras reproductivas, se apreció un útero vesiculado en forma de collar de perlas (Fig. 2a). Una vez extraído el órgano y al realizar una incisión longitudinal, se observó presencia de sangre y abundan- tes formaciones quísticas macroscópicas en el endometrio, a través de toda la extensión de ambos cuernos uterinos (Fig. 2b). Al abrir las bolsas ováricas y examinar los ovarios se encontraron estructuras quísticas de aspecto transparente y un diámetro de entre 10 y 15 $\mathrm{mm}$. La conjunción de los hallazgos clínicos y anatómicos permitió corroborar la presencia de un cuadro concomitante de hematometra e HEQ.

\section{Discusión}

El estudio de las secreciones vaginales de la perra, mediante la técnica de citología vaginal, es una importante herramienta en el diagnóstico de estados fisiológicos y patológicos del tracto genital. El flujo vaginal sanguinolento se relaciona usualmente con el calor (proestro-estro), coagulopatías, laceraciones vaginales, subinvolución de sitios placentarios, infecciones y neoplasias (Fieni, 2006). En el presente caso, los resultados de la colpocitología, con claro predominio de células parabasales e intermedias y ausencia de células superficiales, permitieron concluir que la hembra no se encontraba bajo influencia 
estrogénica y que el cuadro citológico era compatible con anestro. Esto fue corroborado al medirse niveles basales de progesterona sérica $(<1 \mathrm{mg} / \mathrm{ml})$, característico de dicha etapa del ciclo estral (Concannon, 2011).

Dada la posibilidad de que el flujo fuese de origen uterino, se procedió a realizar un estudio ecográfico del órgano, que determinó la presencia de contenido anecoico y aumento de tamaño de los cuernos; situación que permitió establecer los prediagnósticos de piometra o hematometra (Fieni, 2006). Si bien la paciente no presentaba signos clínicos de piometra, tales como anorexia, vómito, polidipsia/poliuria, decaimiento o fiebre, se practicó un hemograma, el cual no mostró la leucocitosis con neutrofilia característica de piometra (Root Kustritz y Barber, 2003: Bigliardi y Pamigiani, 2004); sin embargo, se observó una anemia moderada normocítica normocrómica regenerativa, la que se explicaría por la metrorragia que presentó en las últimas dos semanas.

El estudio posquirúrgico del útero permitió evidenciar la presencia de sangre en el lumen. Según Fieni (2006), las metrorragias en perras no grávidas se pueden observar fuera del periodo de celos, teniendo su origen más común en neoplasias uterinas como el tumor venéreo transmisible o condiciones como hiperestrogenismo iatrogénico o hiperestrogenismo asociado a quistes foliculares funcionales, o tumor ovárico de células de la granulosa. Si bien en el presente caso se observaron quistes ováricos, la ausencia de signos de estrogenización y el valor basal de progesterona sugieren que los quistes no estaban funcionales, dificultando establecer la causal del sangrado uterino.

En relación con las múltiples estructuras quísticas de aspecto seroso observadas en la mucosa uterina, Schlafer (2012) indica que la condición quística más común en el útero de la perra es la HEQ, la cual compromete al epitelio glandular y presenta una gran variación en cuanto a tamaño, número, distri- bución, histomorfología e importancia clínica. Esta condición se describe asociada a la fase luteal del ciclo canino, dada la prolongada influencia de la progesterona (Chen et al., 2001; Schlafer y Gifford, 2008). En el presente caso, el efecto de la progesterona luteal es discutible, ya que se sugiere la ocurrencia de ciclos anovulatorios en perras con historial de IIE cortos ( $<3$ meses) (Meyers-Wallen, 2007).

Una posible explicación para la hematometra y consiguiente metrorragia podría adjudicarse a la extravasación como consecuencia de una rotura vascular originada en cambios proliferativos del endometrio, asociados a una forma de hiperplasia endometrial conocida como hiperplasia endometrial pseudo-placentacional, la cual se caracteriza por una lesión uterina con marcado crecimiento endometrial de características similares a la reacción decidual de la placentación endoteliocorial zonal característica de la especie canina (Nomura y Funahashi, 1999; Schlafer y Gifford, 2008).

Según la literatura revisada, se puede indicar que el caso expuesto resulta atípico, dado que la hembra al momento de manifestar los signos clínicos se encontraba en estado de anestro. Sin embargo, por la irregularidad de sus ciclos estrales, cabría postular que las lesiones uterinas podrían haberse generado en un ciclo anterior, asociadas a una posible funcionalidad de los quistes ováricos observados.

\section{Literatura Citada}

1. Bigliardi E, Pamigiani E. 2004. Ultrasonography and cystic hyperplasiapyometra complex in the bitch. Reprod Dom Anim 39: 136-240.

2. Chen Y, Wright P, Lee C. 2001. A model for the study of cystic endometrial hyperplasia in bitches. J Reprod Fert (Suppl) 57: 407- 414. 
3. Concannon P. 2011. Reproductive cycles of the domestic bitch. Anim Reprod Sci 124: 200-210. doi: 10.1016/ j.anireprosci.2010.08.028

4. Fieni F. 2006. Patología de los ovarios y el útero. En: Wanke M, Gobello C (eds). Reproducción en caninos y felinos domésticos. Buenos Aires: Inter-Médica. p 75-95.

5. Jain N. 1993. Essentials of veterinary hematology. Philadelphia: Lea \& Febiger. $417 \mathrm{p}$.

6. Johnston S, Root Kustritz, M, Olson P. 2001. Disorders of the canine uterus and uterine tubes (oviducts). En: Johnston S, Root Kustritz M, Olson P (eds). Canine and feline theriogenology. USA: WB Saunders. p 206-224.

7. Kempisty B, Bukowska D, Wozna M, Piotrowska H, Jackowska M, Zuraw A, Ciesiolka1 S, et al. 2013. Endometritis and pyometra in bitches: a review. Vet Med Czech 58: 289-297.

8. Meyers-Wallen V. 2007. Unusual and abnormal canine oestrus cycles. Theriogenology 68: 1205-1210. doi: 10.1016/j.theriogenology.2007.08.019

9. Nomura K, Funahashi H. 1999. Histological characteristics of canine deciduoma induced by intrauterine inoculation of $E$. coli suspension. J Vet Med Sci 61: 433-438. doi: 10.1292/ jvms.61.433
10. Pretzer S. 2008. Clinical presentation of canine pyometra and mucometra: a review. Theriogenology 70: 359-363. doi: 10.1016/j.theriogenology.2008.04.028

11. Root Kustritz M, Barber J. 2003. Uterine disorders. En: Root Kustritz M (ed). Small animal theriogenology. USA: Elsevier Science. p 367-394.

12. Schlafer D, Gifford A. 2008. Cystic endometrial hyperplasia, pseudoplacentational endometrial hyperplasia, and other cystic conditions of the canine and feline uterus. Theriogenology 70 : 349-358. DOI: $10.1016 /$ j.theriogenology.2008.04.041

13. Schlafer D. 2012. Diseases of the canine uterus. Reprod Dom Anim 47 (Suppl) 6: 318-322. doi: 10.1111/ rda.12064

14. Tello L, Martín F, Valdés A, Albala A. 1996. Estudio comparativo de signos, ecográficos, radiográficos y postquirúrgicos en 50 perras con piometra. Arch Med Vet 28(1): 137-143.

15. Verstegen J, Dhaliwal G, VerstegenOnclin K. 2008. Mucometra, cystic endometrial hyperplasia, and pyometra in the bitch: advances in treatment and assessment of future reproductive success. Theriogenology 70: 364-274. 10.1016/j.theriogenology.2008.04.036 